\title{
An Analytical Framework for the Assessment of the Mobile IP overhead involved in the Integrated Internet-MANET
}

\author{
doi:10.3991/ijim.v4i1.1027 \\ Khaleel Ur Rahman Khan ${ }^{1}$, Rafi U Zaman ${ }^{1}$ and A. Venugopal Reddy ${ }^{2}$ \\ ${ }^{1}$ M. J. College of Engineering \& Technology, Hyderabad, India \\ ${ }^{2}$ U.C.E, Osmania University, Hyderabad, India
}

\begin{abstract}
Quite a few strategies have been proposed for integrating mobile ad hoc networks and the Internet. A need has been felt for an analytical model that would facilitate a formal definition and comparison of different integration strategies without actually implementing them in a simulated environment. In this paper, we present an analytical framework based on the concept of a Relay Path Set (RPS). This model can be used to describe, compare the different strategies for integrating mobile Ad Hoc networks and the Internet, propose new strategies and suggest improvements in the existing strategies at different module levels. The proposed model has been applied on a few of the existing Integration strategies and performance comparison has also been made. We also discuss the future work that can be carried out using the proposed framework.
\end{abstract}

Index Terms-Keywords: Analytical Model, Integration Strategy, Mobile Ad Hoc Network, Relay Path Set

\section{INTRODUCTION}

Mobile ad hoc network, on its own, provides limited utility. To extend its capability, a mobile ad hoc network is connected to the Internet or a wired network. Many strategies have been proposed for integrating mobile ad hoc networks with the Internet. The challenges and a review of the proposed strategies are discussed in (Khaleel et al., 2008a). While there are many researchers working on the problem of integrating mobile ad hoc networks with the Internet, very few have ventured to compare the different strategies. A framework based on the concept of a Relay Node Set (Tao et al., 2003), is proposed for the comparison of different wireless ad hoc routing protocols. As of now, no analytical model exists for the comparison of strategies for integrating mobile ad hoc network and the Internet.

In this paper, we propose a framework for assessing the performance of the strategies for integrating mobile ad hoc networks with the Internet, which is based on the concept of a Relay Path (RP). We developed an analytical model which is then applied on a few of the strategies. The analytical framework characterizes the Integration strategies using four modules. This model has the following capabilities.

The integration strategies could be formally described by a modular approach, thereby giving a complete insight to the researchers about their working mechanism.
The framework can be used to compare the different strategies with analytical equations.

Modifications and improvements can be proposed at the different modules of the framework possibly by replacing one protocol with another or one strategy/mechanism with another.

The Framework can also facilitate in the proposal of altogether new integration strategies as well.

Presently, in this paper we concentrated on the first three capabilities and we are still working on the other capability. The rest of the paper is organized as follows. Section 2 presents the Background and the previous work. Section 3 presents the proposed Analytical model based on the concept of a Relay Path. Section 4 illustrates the application of the proposed framework on the Integration strategies MIPMANET (Jönsson et al., 2000), Sun's strategy (Sun et al., 2002), Ammari's strategy (Ammari et al., 2004), bi-directional connectivity Framework (Khaleel et al., 2008b), and the Hierarchical approach (Khaleel et al., 2008). Section 5 presents simulation results and compares the three strategies based on our framework. Section 6 discusses the future work and concludes the paper.

\section{BACKGROUND}

Connecting MANETs to the Internet helps build a hybrid network to provide MANET nodes with multi-hop wireless access to the Internet services, anytime and anywhere. The design of this kind of hybrid network faces several challenges. Some of the challenges include the Load balancing of the Gateways (GW), Addressing \& Routing Mechanisms within the MANETs, Security, Service and Gateway Discovery Mechanisms, Avoiding Dead zones and tackling the problem of selfish behaviour of Mobile nodes (Khan et al., 2008a).

In the wireless Internet, the Mobile IPv4 (Perkins 1997) protocol requires that mobile hosts be one hop away from foreign links, i.e. Mobile IP Gateways. In other words, only hosts at the very edges of the wired Internet can move and benefit from the Mobile IP services. It may be noted that the Mobile IP did not take into account the possibility of connecting MANETs to the Internet. Thus, the challenge that faces connecting MANETs to the Internet is extending Mobile IP to manage node mobility even when these nodes are multi-hop away from the edge of the Internet, which is governed by Mobile IP Gateways. Hence, any solution to connecting MANETs to the Internet needs to extend the notion of 'one-hop away' to 
'multi-hop away' to facilitate multi hop wireless communication between MANET nodes and Mobile IP Gateway. Several approaches to connecting MANETs to the Internet exist in the literature (Khan et al., 2008a). They consider Mobile IP Gateways as an interface between MANET and the Internet. These agents run ad hoc routing protocols to connect to MANETs and use an updated version of Mobile IP to connect to the Internet. Few strategies use Mobile IPv4 and others are based on Mobile IPv6 (Johnson et al., 2004).

\section{A. Existing Integration Strategies}

Few of the existing integration strategies are summarized below.

- Lei and Perkins (Lei et al., 1997) proposal for integrating ad hoc Networks and Internet, makes use of a Routing Information Protocol (RIP) within the ad hoc network.

- Broch and Johnson (Broch et al., 1999) proposed a solution to the integration of MANET with Mobile IP. It makes use of Border Gateway with two interfaces. One is configured with the normal IP, which is connected to the Internet. While the other connected to the MANET uses DSR protocol to route packets within MANET.

- Sun's proposal (Sun et al., 2002) makes use of AODV (Perkins et al 2002) and Mobile IP to enable Internet connectivity to Mobile nodes. AODV is used for routing within the MANET, while Mobile IP is used for assigning care of address to the Mobile nodes. Hand off occurs only if a mobile node has not heard for more than one beacon interval.

- Ratanchandani and Kravets (Ratanchandani et al., 2003), has given a hybrid scheme to provide Internet connectivity to the MANET nodes, using Mobile IP. The scheme uses techniques such as TTL scoping of Agent advertisements, eavesdropping and caching Agent advertisements to combine the advantages of proactive and reactive approaches to providing connectivity.

- Tseng (Tseng et al., 2003) proposal of the Integration and Implementation is based on IEEE $802.11 \mathrm{~b}$ wireless LANs. Issues like overlapping of MANETs, dynamic adjustment of mobile Agent's service coverage's, support of local broadcast and various communication scenarios are addressed.

- Ammari (Ammari et al., 2004) propose a three tier architecture which provides Internet connectivity for mobile nodes. The first tier consists of the Internet Gateways, the second tier consists of mobile gateways and the third tier consists of mobile nodes which desire Internet connectivity. The main advantage of this scheme is it is scalable due to the use of mobile gateways.

\section{ANALYTICAL FRAMEWORK}

\section{A. Definitions}

1) Path: Consider an undirected graph $G$ with the set of vertices $\mathrm{V}$ and the set of edges $\mathrm{E}$. A path is a sequence of vertices and edges of the form A-B-C-D, where A,B,C,D are vertices and (A-B) (B-C) are edges.

2) Relay Node $(\mathrm{RN})$ : In a mobile ad hoc network, a node that has the capability to retransmit control messages is called a relay node. Non-Relay nodes always stay silent after they have processed control messages broadcast by their neighbours.

3) Relay Path (RP): A Relay Path is a path which contains only Relay Nodes as its vertices.

4) Relay Path Set (RPS): More than one Relay Path may exist between a pair of nodes in a mobile ad hoc network. The set of all such Relay Paths existing between a pair of nodes is called the Relay Path Set of that pair of nodes.

Let us consider the graph in Figure 1, It depicts a mobile ad hoc network and the solid lines represent connectivity between mobile nodes. Let mobile nodes A, B, E and $F$ be relay nodes and the rest of the nodes be nonrelay nodes. Then A-B-E is a relay path. A-B-C-D is not a relay path even though it is a path, since $C$ is not a Relay Node. The Relay Path Set for the pair of nodes $(A, E)$ is $\{$ A-B-E, A-E $\}$.

\section{B. General Architecture of Mobile IPv4 based Integration Strategy}

Mobile IP provides the basis for a mobile node to access Internet connectivity. But, the problem with it is that it provides only one hop connectivity. To overcome this drawback, many integration strategies work around Mobile IP to provide multi hop Internet connectivity for a mobile ad hoc network. The general architecture of an integration strategy is depicted in Figure 2.

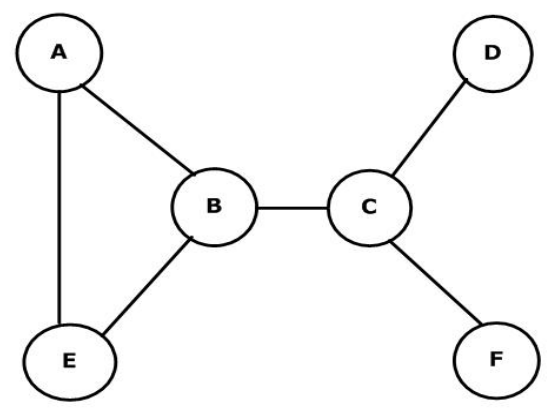

Figure 1. Example of Relay Path Set

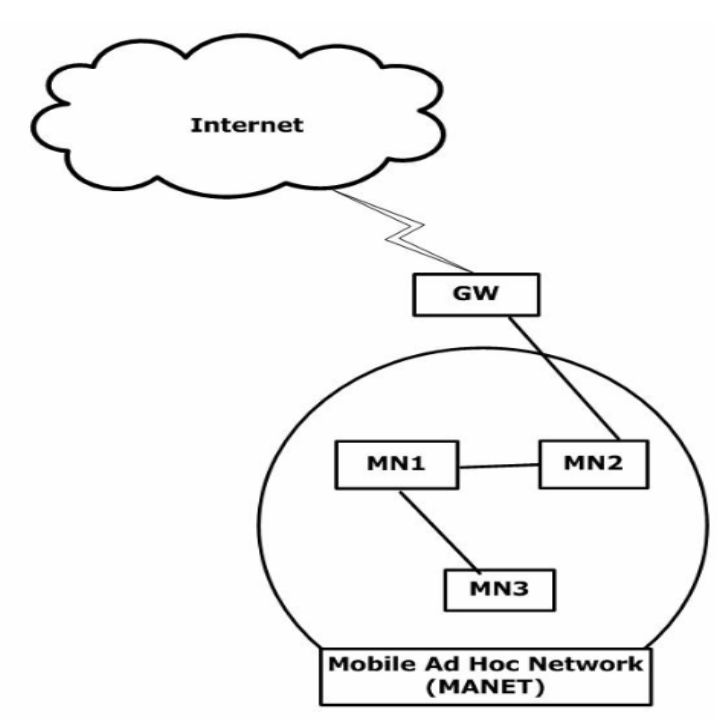

Figure 2. General Architecture of Integration Strategy 
In Figure 2, the solid lines represent wireless connectivity. GW represents the Internet Gateway. Next to the $\mathrm{GW}$, we have a mobile ad hoc network of three nodes. Mobile node MN2 can get wireless Internet access via $\mathrm{GW}$, since it is one hop away. The general problem of an integration strategy is to provide Internet connectivity to those mobile nodes which are multi hop away from the GW, in our case, mobile nodes MN1 and MN3. Many integration strategies use the nodes which are one hop away from the GW as intermediate nodes to provide internet connectivity to those nodes which are multi hop away from the GW.

To get Internet access, a mobile node has to register itself with the GW. Based on how this registration is done, integration strategies are classified as Proactive or Reactive. In a proactive mechanism, the GW periodically sends out agent advertisements, which contain its address. Mobile nodes can use these agent advertisements to register with the GW. In a reactive mechanism, a mobile node sends an agent solicitation message to a $\mathrm{GW}$, in order to obtain Internet connectivity

\section{Proposed Framework}

The proposed framework is built around the concept of the Relay Path. The framework contains four modules, as shown in Figure 3. The arrows imply the dependency of one module on the other. For example, the Handover Module is dependent on the Discovery Module as well as the Registration Module.

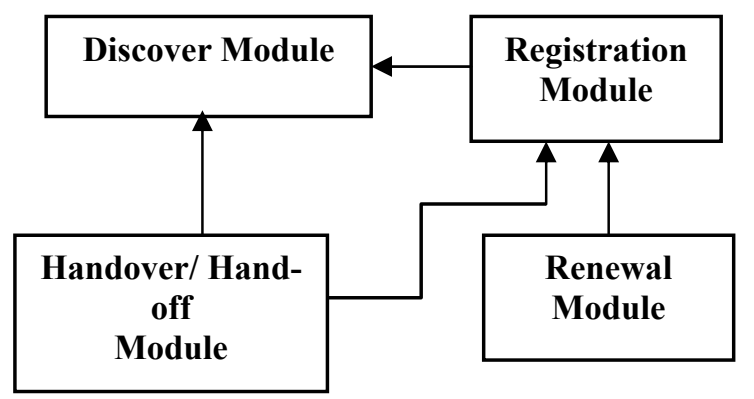

Figure 3. The Four Module Framework for the Integration Strategy

\section{1) Discover Module}

Refers to the search for a Gateway (GW) by a Mobile Node. This discovery mechanism is aided by periodic Agent Advertisements in a proactive integration scheme and Agent Solicitations in the case of reactive approach of the discovery of the Gateway.

In the case of a proactive approach, the Gateway periodically broadcasts Agent Advertisements into the MANET. These Agent Advertisements are received by mobile nodes which are one hop away from the Gateway. If a mobile node desires Internet connectivity, it sends a registration request to the Gateway (this process will be covered in the Registration Module). If not, it just ignores this Agent Advertisement. In either case, it forwards this Agent Advertisement to its neighbors. If a mobile node is multiple hops away from the GW, then the Agent Advertisement will traverse the intermediate nodes in order to reach the mobile node. The set of nodes that the Agent advertisement traverses before reaching the mobile node forms one Relay Path (RP) from the Gateway to the mobile node. The same Agent Advertisement may have traversed multiple paths to reach the same mobile node.
Therefore, more than one RP may exist between a pair of Gateway and mobile node. The set of all Relay Paths that exist between a pair of Gateway and mobile node for a particular Agent Advertisement is called the Relay Path Set. The discover module is concerned with discovering the Relay Path Set of every Gateway-Mobile Node pair in the MANET.

In the case of a reactive approach, the Gateway does not broadcast Agent Advertisements into the MANET. If a mobile node desires Internet connectivity, it broadcasts an Agent Solicitation message. When a Gateway receives this Agent Solicitation message, it unicasts its own Agent Advertisement message to the mobile node. The set of nodes that the Agent Advertisement traverses before reaching the mobile node will be one Relay Path. If there is more than one Relay Path, that is, if the same Agent Advertisement has traversed multiple Relay Paths, then the set of all the Relay Paths is called the Relay Path Set. As in the proactive approach, the discover module is concerned with discovering the Relay Path Set of every Gateway-Mobile Node pair in the MANET.

\section{2) Registration Module}

After the execution of the Discover Module, either through the proactive approach or reactive approach, a mobile node will have the knowledge about all the reachable Gateways. Next, the mobile node desiring Internet connectivity has to select one Gateway to register with. This Gateway selection mechanism is dependent on the particular strategy and is generally dependent on metrics like hop count or physical distance or a combination of various metrics. After the Gateway selection is done, the mobile node unicasts a Registration Request to the selected Gateway. For this purpose, one Relay Path from among the available paths in the RPS is used. The Gateway responds by sending a Registration Reply. This completes the registration process.

\section{3) Renewal Module}

The registration of a mobile node with the GW is not valid indefinitely. In order to take into account the mobility of the nodes, the mobile nodes are required to periodically re-register with their respective GW. The Registration Renewal interval varies from strategy to strategy. If it is too low, then there will be a lot of registration renewals. If it is too high, then Data Packets may be lost due to stale routes. The Relay Path used in the registration module may also be used for the purpose of renewal, or a different relay path from the relay path set may be used. This job is handled by the Renewal Module.

\section{4) Handover/Handoff Module}

If a mobile node fails to renew its registration with its GW for a specified length of time, the GW assumes that it has moved out of its coverage area. The mobile node registers itself with another GW. This is called a cell switch and is handled by the Handover Module. The Handoff process has three phases (Jing et al, 2007).

The first phase known as the detection is the discovery of the need for handover. This is dependent on the Integration strategy's Handoff algorithm. For example, MIPMANET (Jonsson et al, 2007) uses a Handoff algorithm called MIPMANET Cell Switching Algorithm. If the need for handoff is justified, then the second phase is activated. 
The second phase, called search covers the acquisition of the information needed to perform the handover. This involves the discovery of an alternate Gateway. Therefore, the Discover module is activated. At the end of the Discover module, the mobile node has the necessary information which is needed to perform the handoff.

Finally the handover is performed during the third phase which is the execution. Execution of the handover is nothing but registering with the newly discovered Gateway. Hence the Registration module is executed.

The main driver of the integration strategies is the amount of control information in the form of MIP control packets, which is exchanged in the network. More reliable strategies require frequent exchange of control information, in turn increasing the control overhead. Control information can be agent advertisement messages, registration reply messages, agent solicitation messages or any other kind of messages. Using the above framework, we develop an analytical model which represents the total control overhead of an integration strategy. The total control overhead of an integration strategy is the sum of all the control messages involved in the above four modules. Equation (1) gives the total control overhead of an Integration Strategy.

Total Control Overhead, $\mathrm{O}_{\text {IS }}$ of Integration Strategy (IS) is given by:

$$
\begin{aligned}
& \mathrm{O}_{\text {IS }}=\mathrm{O}_{\text {discover }}+\mathrm{O}_{\text {register }}+\mathrm{O}_{\text {renewal }}+\mathrm{O}_{\text {handover }} \\
& \text { Where, } \\
& \mathrm{O}_{\text {discover }}=\sum \mathrm{N}_{\text {discover }} \sum \mathrm{S}_{\mathrm{RPS}} \mathrm{P}_{\text {discover }} \\
& \mathrm{O}_{\text {register }}=\sum \mathrm{N}_{\text {register }} \sum \mathrm{S}_{\text {register }} \mathrm{P}_{\text {register }} \\
& \mathrm{O}_{\text {renewal }}=\sum \mathrm{N}_{\text {renewal }} \sum \mathrm{S}_{\text {renewal }} \mathrm{P}_{\text {renewal }} \\
& \mathrm{O}_{\text {handover }}=\mathrm{O}_{\text {detection }}+\mathrm{O}_{\text {execution }}
\end{aligned}
$$

Where O stands for Overhead, $\mathrm{P}$ is the control packet size, $\mathrm{S}$ is the size of the relay path set, $\mathrm{N}$ represents the number of control operations that take place in time period ' $t$ ', and the subscripts represent the names of the respective modules associated variables.

$\mathrm{O}_{\text {discover }}$ is the overhead of discovery of a gateway by a mobile node. $\mathrm{O}_{\text {register }}$ is the overhead of registering the mobile node with the gateway. Every registration needs to be renewed at periodic intervals. $\mathrm{O}_{\text {renewal }}$ is the overhead of renewing a registration. $\mathrm{O}_{\text {handover }}$ is the overhead of switching from one gateway to another. This is dependent on two components, the overhead of detecting the need for a handover expressed as $\mathrm{O}_{\text {detection }}$ and the overhead of actually executing the handover, expressed as $\mathrm{O}_{\text {execution }} . \mathrm{N}_{\text {dis- }}$ cover, $\mathrm{N}_{\text {register, }}$ and $\mathrm{N}_{\text {renewal }}$ are the number of control operations taking place in the Discover, Register and Renewal modules respectively. $\mathrm{S}_{\mathrm{RPS}}$ is the size of all the Relay Paths discovered in the discover module, $\mathrm{S}_{\text {register }}$ and $\mathrm{S}_{\text {re- }}$ newal are the sizes of the RP of the register and renewal modules respectively. $\mathrm{P}_{\text {discover }}, \mathrm{P}_{\text {register }}, \mathrm{P}_{\text {renewal }}$ are the control packet sizes of the Discover, Register and Renewal modules respectively.

The robustness of the Integration strategy is dependent on the overhead associated with the Handover module. Less this overhead, more robust and reliable is the strategy. By and large, if one of the terms becomes less, some of the other terms may become more. Proactive approach of registration has generally more overhead than the reactive approaches. The developers and the designers should try to balance these factors so that the strategy should re- sult in producing overall optimal overhead. The description of the Integration Strategies by this Analytical Framework can help the researchers to find the various Overhead factors involved. Later, the comparison of results among the strategies in this framework can be used to improve the strategies and even propose new approaches. It may be noted that the same framework can be used for the IPv4 and IPv6 based strategies.

\section{APPLYING THE ANALYTICAL FRAMEWORK ON FEW OF THE INTEGRATION STRATEGIES}

Our proposed model is applied on MIPMANET, Sun et al's strategy, Ammari et al's strategy, the Full Duplex connectivity framework and the Hierarchical approach of integrating MANET and the Internet.

\section{A. MIPMANET Strategy}

1) Description of MIPMANET: MIPMANET (Jonsson et al, 2007) uses ad hoc On-Demand Distance Vector (AODV) protocol for routing within the ad hoc network. The protocol works as follows: Mobile Nodes within an ad hoc network that want Internet access use their home address for all communication and register with a Gateway. To send a packet to a host on the Internet, the packet is tunneled to the Gateway with which the mobile node is registered with. To receive packets from hosts on the Internet, the packet is routed to the gateway by ordinary Mobile IP mechanisms. The gateway will then deliver the packets to the node in the ad hoc network. Nodes that do not require Internet access see the ad hoc network as a stand alone network and they do not need any knowledge about routes to destinations outside the ad hoc network. A conceptual view of MIPMANET at different levels of the protocol hierarchy is depicted in Figure 4. The gateway acts as an Internet gateway.

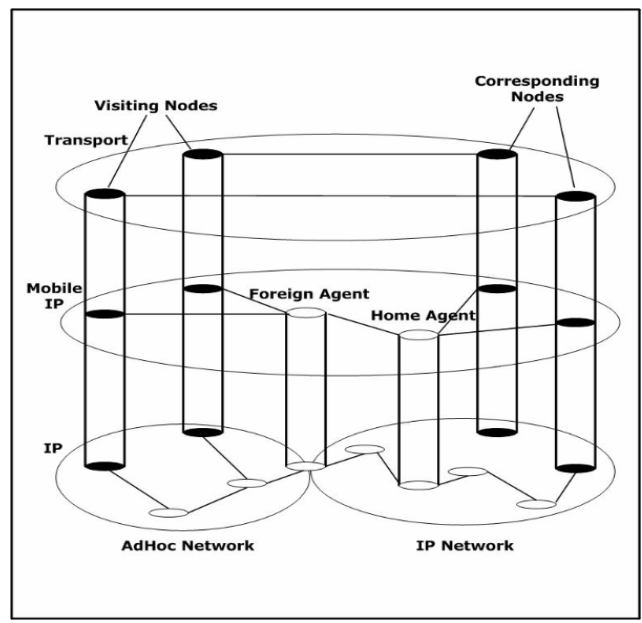

Figure 4. A conceptual view of MIPMANET

\section{B. Analyzing MIPMANET}

$\mathrm{O}_{\text {MIPMANET }}=$ Sum of the overheads of the modules as stated in Equation 1.

$\mathrm{O}_{\text {discover: }}$ MIPMANET uses a proactive gateway discovery approach wherein the GW periodically floods the ad hoc network with agent advertisement packets $\mathrm{P}_{\text {AA }}$. The discover module of MIPMANET is given by the following equation: 


$$
\mathbf{O}_{\text {discover }}=\sum_{\mathbf{i}=\mathbf{1}} \sum_{\mathbf{j}=\mathbf{1}} \mathbf{P}_{\text {AA }}
$$

Where, Si,RPS is the set of RP that connect the ith pair of GW and mobile node (MN).

$\mathrm{P}_{\mathrm{AA}}$ is the size of the Agent advertisement packet.

NGM is the set of all Gateway-Mobile Node pairs in the ad hoc network.

\section{$\mathrm{Si}, \mathrm{RPS}$}

$\sum$ represents the summation of agent advertisement packets $\mathrm{j}=1$

$\mathrm{P}_{\mathrm{AA}}$ over the set RPS that exists between the ith pair of GW and MN. For example, consider Figure5. The Figure shows a mobile ad hoc network with three mobile nodes $\mathrm{MN} 1, \mathrm{MN} 2$ and MN3, and the integration of this mobile ad hoc network with the Internet through the gateway GW. MN1 and MN2 are one hop away from GW and MN3 is two hops away from the GW. MN3 can access the GW either through MN1 or MN2, in order to access the Internet. Let the GW-MN3 pair be the $\mathrm{i}^{\text {th }}$ pair of $\mathrm{GW}$ and $\mathrm{MN}$. Then, the set of all RP that exist between the GWMN3 pair is $S_{i, R P S}=\{\{G W, M N 1, M N 3\},\{G W, M N 2$, MN3 $\}\}$, where $\{\mathrm{GW}, \mathrm{MN} 1, \mathrm{MN} 3\}$ and $\{\mathrm{GW}, \mathrm{MN} 2$, MN3 3 are the only two Relay Paths that exist between GW and MN3. MN1 and MN2 are in the neighbourhood of GW. When GW floods the MANET with agent advertisement packets, these two nodes are the only ones which receive them. They in turn forward these packets to their neighbours, which in our case is the common neighbour MN3. Therefore the total number of control packets required for MN3 to discover GW is 4 (Two each for each RP).

\section{NGM}

Finally, $\sum$ represents the summation of all such GW-MN $\mathrm{i}=1$

pairs that exist in the ad hoc network and NGM is the number of all GW-MN pairs that exist in the ad hoc network. Therefore, $\mathrm{O}_{\text {discover }}$ represents the total control overhead in terms of the agent advertisement packets required so that each mobile node in the ad hoc network discovers every gateway.

$\mathrm{O}_{\text {register: }}$ The agent advertisement contains the source address of the GW. Once a mobile node gets an agent advertisement, it can find a path to the GW and register with the GW. In MIPMANET, AODV is used for routing within the MANET as well as to find a path to the MANET. Once this path is established, all data packets are tunnelled to the GW using the same path. In terms of the proposed analytical model, finding a path is nothing but selection of one of the RP from the RPS discovered in the discover module. The selection of this RP is dependent on the underlying routing protocol, in this case, AODV. In the worst case, the longest RP is selected.

Let the selected RP for the ith pair of GW-MN be $\mathrm{S}_{\mathrm{i} \text {,register }} \cdot \mathrm{S}_{\mathrm{i} \text {,register }}$ is the subset of $\mathrm{S}_{\mathrm{i}, \mathrm{RPS}}$ Then, $\mathrm{O}_{\text {register }}$ is given by,

$$
\begin{aligned}
& \text { NGM Si,register } \\
& \mathrm{O}_{\text {register }}=\sum_{\mathrm{i}=1} \\
& \sum_{j=1} \quad\left[\begin{array}{l}
\text { PREG_REQ }_{\text {REG }}+ \\
\left.P_{\text {REG_REP }}\right]
\end{array}\right.
\end{aligned}
$$

Where, Si,register is the size of the RP selected to register with the gateway.

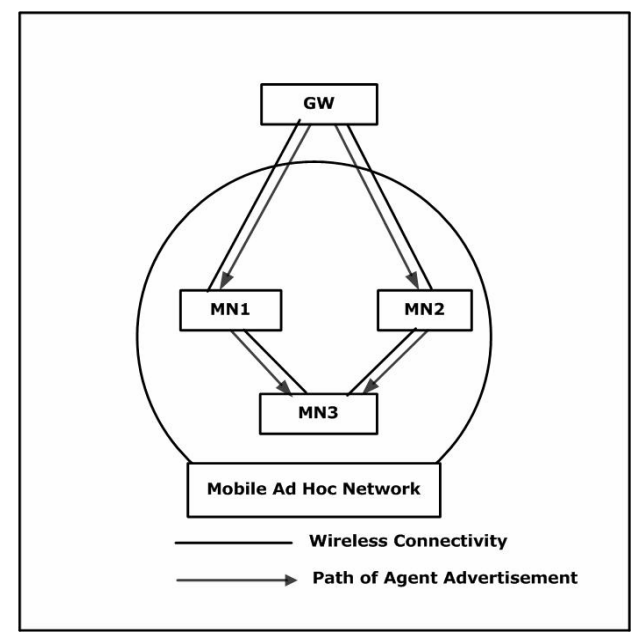

Figure 5. GW floods the ad hoc network with agent advertisements.

$P_{\text {REG_REQ }}$ and $P_{\text {REG_REP }}$ are the sizes of the Registration Request and Registration Reply packets respectively.

NGM is the set of all Gateway-Mobile Node pairs in the ad hoc network.

In our example, there are two candidate RP i.e., $\{\mathrm{GW}$, $\mathrm{MN} 1, \mathrm{MN} 3\}$ and $\{\mathrm{GW}, \mathrm{MN} 2, \mathrm{MN} 3\}$. Let us say AODV selects $\{\mathrm{GW}, \mathrm{MN} 1, \mathrm{MN} 3\}$ as the relay path between $\mathrm{GW}$ and $\mathrm{MN} 3$. Then, MN3 sends a registration request packet to GW. On successful registration, GW sends back a registration reply packet. Again the registration overhead is calculated for all the pairs of GW-MN, NGM. P REG_REQ $_{\text {R }}$ and $P_{\text {REG REP }}$ are the registration request and registration reply packet sizes respectively.

$\mathrm{O}_{\text {renewal }}$ : MIPMANET requires that the registration of a mobile node with a gateway be renewed at regular, preset intervals, called beacons. The registration process may use the same RP as $\mathrm{S}_{\mathrm{i} \text {,register }}$ or it may use another RP to renew its registration with the same $\mathrm{GW}$. In the above example, we have assumed that $\mathrm{S}_{\mathrm{i}, \text { register }}$ is $\{\mathrm{GW}, \mathrm{MN} 1, \mathrm{MN} 3\}$, whereas for renewal, $\{\mathrm{GW}, \mathrm{MN} 1, \mathrm{MN} 3\}$ may be used or $\{\mathrm{GW}, \mathrm{MN} 2, \mathrm{MN} 3\}$ may be used. In either case, we call the RP used to renew the registration as $\mathrm{S}_{\mathrm{i}, \text { renewal }} . \mathrm{S}_{\mathrm{i} \text {,renewal }}$ is also the subset of $S_{i, R P S}$ and $S_{i, \text { renewal }}$ may or may not be equal

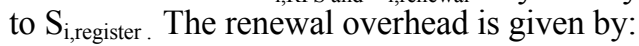

$$
\begin{array}{ccc}
\text { NGM } & \multicolumn{2}{c}{\text { Si, renewal }} \\
\mathrm{O}_{\text {renewal }}=\sum_{\mathrm{i}=1} & \sum_{\mathrm{j}=1}\left[_{\mathrm{P}_{\text {REG_REQ }}+}^{+} \mathrm{P}_{\text {REG_REP }}\right]
\end{array}
$$

Where, NGM, $\mathrm{P}_{\text {REG_REQ }}, \mathrm{P}_{\text {REG_REP }}$ have the same meaning as in the registration module. $\mathrm{S}_{\mathrm{i} \text {,renewal }}$ is the size of the RP selected to renew the registration with the gateway.

$\mathrm{O}_{\text {handover: }}$ In MIPMANET, a handover can occur under two cases. One, when a mobile node moves out of the range of the gateway with which it is currently registered with. Two, when a mobile node stays with its currently registered gateway, but receives two consecutive agent advertisements from another gateway which is at least two hops closer than the current gateway (MIPMANET Cell Switching Algorithm). According to our framework, the overhead for handover depends on two components, de- 
tecting the need for handover, and finally, executing the handover.

Let $S_{\text {new,RP }}$ and $S_{\text {old,RP }}$ be the size of the Relay Paths of the mobile node with respect to the new and old gateways. $\mathrm{O}_{\text {detection }}$ can be expressed by the following equation.

$$
\mathrm{S}_{\text {new }, \mathrm{RP}}+(\mathrm{a}+2)=\mathrm{S}_{\text {old }, \mathrm{RP}}
$$

Where,

$S_{\text {new,RP }}$ and $S_{\text {old,RP }}$ are the sizes of the new and old RP respectively. ' $a$ ' is an integer constant, whose final value is the difference between the sizes of the old and new RNS. For example, if the $S_{\text {old, RP }}$ is 10 and $S_{\text {new, RP }}$ is 8 , then, $a=$ 0 .

$\mathrm{O}_{\text {execution }}$ is nothing but $\mathrm{O}_{\text {register }}$ with the new gateway. The handover is actually executed when following condition holds true for two consecutive agent advertisements.

$$
\left(S_{\text {new }, R P}+2\right) \leq S_{\text {old,RP }}
$$

\section{Sun's Integration Strategy}

\section{1) Overview of Sun's Strategy (Sun et al, 2002)}

This strategy also uses AODV for routing within the ad hoc network. The protocol is as follows: gateways periodically broadcast Agent advertisements. On receiving an Agent advertisement, a mobile node makes an entry in its Mobile IP Gateway List, which contains the list of Agent advertisements received so far. If the mobile node requires Internet connectivity, it sends a Registration request to the gateway which has sent the Agent advertisement. Registration proceeds as specified by Mobile IP. After processing the Agent advertisement, the mobile node rebroadcasts the packet on its interfaces. This allows mobile nodes which are not within direct transmission range of the gateway to receive Agent advertisements. On the other hand, if a mobile node requires Internet connectivity immediately and no Agent advertisements are forthcoming, it initiates a gateway discovery process by issuing a RREQ message of the AODV protocol which is addressed to the All Mobility Agents multicast group. Therefore, a hybrid approach is used for discovering a gateway.In the event of a neighboring node not supporting Mobile IP, or not having a current route to the gateway, it simply rebroadcasts the request. After registration is complete, the gateway sends a Registration Reply back to the source node. Once the mobile node receives the Registration Reply, it makes an entry in its Gateway List, thereby signifying its registration with the gateway. In order to decide when to switch from one gateway to another, a modified version of the MIPMANET Cell Switching algorithm is used.

\section{2) Analyzing Sun's Integration Strategy} in

$\mathrm{O}_{\mathrm{SUN}}=$ Sum of the overheads of the modules as stated

\section{Equation 1.}

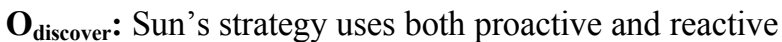
approaches for gateway discovery. The proactive approach is exactly similar to the proactive approach of MIPMANET. In the case where a mobile node requires immediate Internet connectivity and no agent advertisements are forthcoming, the mobile node initiates gateway discovery by broadcasting an agent solicitation message. Therefore,

$$
\mathrm{O}_{\text {discover }}=\mathrm{O}_{\text {proactive }}+\mathrm{O}_{\text {reactive }}
$$

Where,

$$
\begin{array}{lll}
\mathrm{O}_{\text {proactive }}= & \mathrm{NGM} & \mathrm{S}_{\mathrm{i}, \mathrm{RPS}} \\
& \sum_{\mathrm{i}=1} \sum_{\mathrm{j}=1} \mathrm{P}_{\mathrm{AA}} \\
\mathrm{O}_{\text {reactive }}= & \mathrm{NGM} & \mathrm{S}_{\mathrm{i}, \mathrm{RPS}} \\
& \sum_{\mathrm{i}=1} & \sum_{\mathrm{j}=1} \mathrm{P}_{\mathrm{SOL}}
\end{array}
$$

NGM, $\mathrm{S}_{\mathrm{i}, \mathrm{RPS}}, \mathrm{P}_{\mathrm{AA}}$ have the same meaning as in equation (3). $\mathrm{P}_{\mathrm{SOL}}$ refers to the agent solicitation packet size.

In equations (9) and (10), $\mathrm{O}_{\text {proactive }}$ and $\mathrm{O}_{\text {reactive }}$ refer to the overheads involved in the proactive and reactive approaches respectively.

$\mathbf{O}_{\text {register and }} \mathbf{O}_{\text {renewal }}$ : The registration and renewal procedures and semantics of Sun et al's strategy are exactly similar to that of MIPMANET. Therefore,

$$
\begin{aligned}
\mathrm{O}_{\text {register }}= & \sum_{\mathrm{i}=1} \sum_{\mathrm{j}=1}^{\text {NGM Si, register }}\left[\begin{array}{l}
\mathrm{P}_{\text {REG_REQ }}+ \\
\left.\mathrm{P}_{\text {REG_REP }}\right]
\end{array}\right. \\
\mathrm{O}_{\text {renewal }}= & \sum_{\mathrm{i}=1} \sum_{\mathrm{j}=1}\left[\begin{array}{l}
\mathrm{P}_{\text {REG_REQ }}+ \\
\left.\mathrm{P}_{\text {REG_REP }}\right]
\end{array}\right.
\end{aligned}
$$

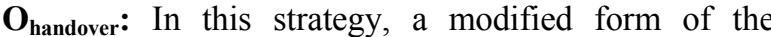
MIPMANET Cell Switching algorithm is used. The requirement of two consecutive agent advertisements is discarded. A mobile node can register with another gateway when it doesn't hear from its gateway for one beacon interval and the new gateway is two hops closer than the old gateway. The following condition must hold true for one beacon interval. Again, $\mathrm{O}_{\text {handover }}$ is the sum of $\mathrm{O}_{\text {detection }}$ and $\mathrm{O}_{\text {execution }}$. $\mathrm{O}_{\text {detection }}$ can be expressed by the following equation.

$$
\mathrm{S}_{\text {new }, \mathrm{RP}}+(\mathrm{a}+2)=\mathrm{S}_{\text {old, } R P}
$$

$\mathrm{O}_{\text {execution }}$ is nothing but $\mathrm{O}_{\text {register }}$ with the new gateway. The handover is actually executed when following condition holds true for one agent advertisement.

$$
\left(\mathrm{S}_{\text {new }, \mathrm{RP}}+2\right) \leq \mathrm{S}_{\text {old,RP }}
$$

\section{Ammari's Three-Tier Strategy}

\section{1) Description of Strategy}

Ammari (Ammari et al., 2004) use a three layered approach, as shown in Figure 6, to provide Internet connectivity in a MANET. The innermost layer contains the wired backbone together with fixed routers, base stations and the Mobile IP Foreign Agents/ Internet Gateway, which will provide Internet connectivity to all the mobile nodes attached to them. The middle layer contains the mobile gateways and mobile Internet nodes which are one-hop away from the elements of the first layer.

The outer layer includes the rest of MANET nodes and visiting mobile Internet nodes, which have lost connectivity with their home networks and want to get connected to them through mobile gateways. The DSDV protocol is used for routing within the MANET. The integration framework considers using some border MANET nodes to connect the rest of MANET nodes to the Internet. These specific MANET nodes are referred as mobile gateways. 


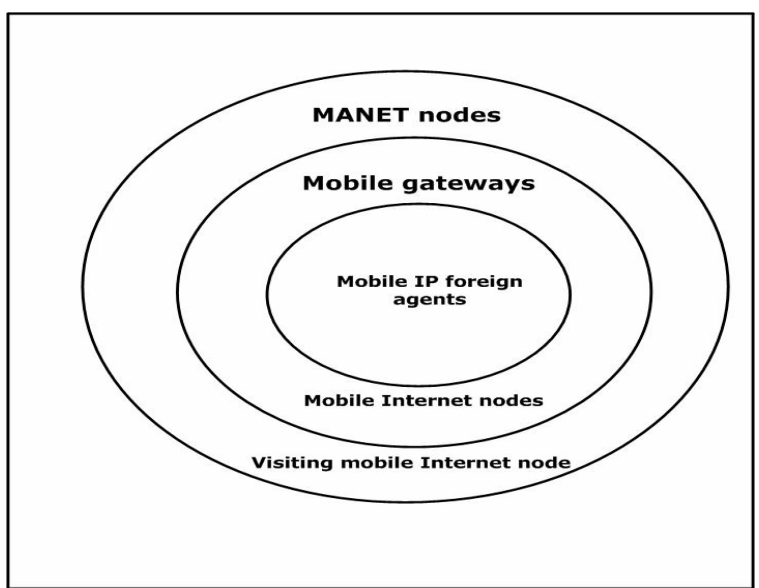

Figure 6. Three Layer Architecture

At least one mobile gateway should exist at every moment. Mobile gateways should be close to foreign agents in order to connect MANET nodes to the Internet through these foreign agents.

A mobile gateway acts as a foreign agent for visiting mobile Internet nodes as well as MANET nodes (henceforth, both are called MANET nodes). Internet connectivity provided to MANET nodes can only be assured through mobile gateways, which can be viewed as dedicated MANET nodes acting as a bridge between the MANET and the Internet. Foreign agents on the Internet willing to serve, broadcast Agent advertisements will be received by mobile gateways. In response, the mobile gateway unicasts a foreign agent registration request to the corresponding foreign agent. Similarly, mobile gateways, which are registered with a foreign agent, will broadcast their Agent advertisements. Due to these Agent advertisements, MANET nodes wishing to have Internet connectivity become aware of the presence of those mobile gateways willing to offer Internet access. Only mobile Internet nodes and mobile gateways can send Agent solicitations to foreign agents. MANET nodes have to send their solicitations only to mobile gateways. Hence, a restricted hybrid approach is used to perform mobile node registration. It is assumed that all MANET nodes, including mobile gateways have valid routable IP addresses.

2) Analyzing Ammari's Integration Strategy

$\mathrm{O}_{\text {AMMARI }}=$ Sum of the overheads of the modules as stated in Equation (1)

O $_{\text {discover: }}$ Gateway discovery is of two types. Type one is the discovery of Internet Gateways by Mobile Gateways and type two is the discovery of Mobile Gateways by mobile nodes. Therefore, the discovery overhead is divided into two sub components, the overhead of discovering the Internet Gateway $\left(\mathrm{O}_{\text {discover IG }}\right)$ and the overhead of discovering the Mobile Gateway $\left(\mathrm{O}_{\text {discover_MG }}\right)$. The total discovery overhead is given by:-

$$
\mathrm{O}_{\text {discover }}=\mathrm{O}_{\text {discover_IG }}+\mathrm{O}_{\text {discover_MG }}
$$

Ammari's strategy uses a hybrid approach for gateway discovery, which is a combination of both proactive and reactive approaches. $\mathrm{O}_{\text {discover IG }}$ and $\mathrm{O}_{\text {discover_MG }}$ are again subdivided into a combination of proactive and reactive overheads. Therefore,

$$
\begin{aligned}
& \mathrm{O}_{\text {discover_IG }}=\mathrm{O}_{\text {proactive }}+\mathrm{O}_{\text {reactive }} \\
& \mathrm{O}_{\text {discover_MG }}=\mathrm{O}_{\text {proactive }}+\mathrm{O}_{\text {reactive }}
\end{aligned}
$$

Where,

$$
\begin{array}{cl}
\mathrm{O}_{\text {proactive }}=\sum_{\mathrm{i}=1} & \sum_{\mathrm{j}=1}^{\mathrm{NGM}} \\
& \mathrm{S}_{\mathrm{AA}} \\
\mathrm{NGM} & \mathrm{S}_{\mathrm{i}, \mathrm{RPS}} \\
\mathrm{O}_{\text {reactive }}=\sum & \sum_{\mathrm{i}=1} \mathrm{P}_{\mathrm{SOL}} \\
\mathrm{j}=1
\end{array}
$$

NGM, $\mathrm{S}_{\mathrm{i}, \mathrm{RPS}}, \mathrm{P}_{\mathrm{AA}}$ have the same meaning as in equation (3). $P_{S O L}$ refers to the agent solicitation packet size. In equations (9) and (10), $\mathrm{O}_{\text {proactive }}$ and $\mathrm{O}_{\text {reactive }}$ refer to the overheads involved in the proactive and reactive approaches respectively.

$\mathbf{O}_{\text {register: Registration is also of two types. Type one is }}$ the registration of Mobile Gateways with the Internet Gateway and type two is the registration of mobile nodes with Mobile Gateways. Therefore, the registration overhead is given by:

$$
\mathrm{O}_{\text {register }}=\mathrm{O}_{\text {register_IG }}+\mathrm{O}_{\text {register_MG }}
$$

Where, $\mathrm{O}_{\text {register_IG }}$ and $\mathrm{O}_{\text {register_MG }}$ are the respective overheads of registering with the Internet Gateway and Mobile Gateway.

$$
\begin{aligned}
& \text { NIMG Si,register }
\end{aligned}
$$

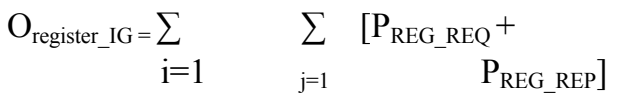

$$
\begin{aligned}
& \text { NMGM Si,register }
\end{aligned}
$$

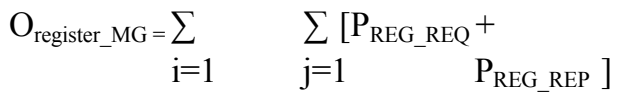

Where NIMG is the number of Internet GatewayMobile Gateway pairs, and NMGM is the number of Mobile Gateway-Mobile Node pairs in the ad hoc network.

O $_{\text {renewal: }}$ The renewal process is again similar to the registration process, the only difference being that the $\mathrm{Si}$,register may or may not be the same as Si,renewal .

$$
\mathrm{O}_{\text {renewal }}=\mathrm{O}_{\text {renewal_IG }}+\mathrm{O}_{\text {renewal_MG }}
$$

Where, $\mathrm{O}_{\text {renewal IG }}$ and $\mathrm{O}_{\text {renewal_MG }}$ are the overhead of renewal with the Internet Gateway and Mobile Gateway respectively.

$$
\begin{aligned}
& \text { NIMG Si, renewal } \\
& \mathrm{O}_{\text {renewal_IG }}=\sum_{\mathrm{i}=1} \quad \sum_{\mathrm{j}=1}\left[\begin{array}{r}
\mathrm{P}_{\text {REG_REQ }}+ \\
\left.\mathrm{P}_{\text {REG_REP }}\right]
\end{array}\right. \\
& \text { NMGM Si,renewal } \\
& \mathrm{O}_{\text {renewal_MG }}=\sum_{\mathrm{i}=1} \quad \sum_{j=1}\left[\mathrm{P}_{\text {REG_REQ }}+\right.
\end{aligned}
$$

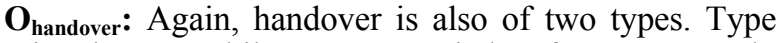
one is when a mobile gateway switches from a currently registered Internet Gateway to another Internet Gateway. 
Type two is when a mobile node switches from a currently registered mobile gateway to another.

$$
\mathrm{O}_{\text {handover }}=\mathrm{O}_{\text {handover_MG }}+\mathrm{O}_{\text {handover_MN }}
$$

Where, $\mathrm{O}_{\text {handover } M G}$ and $\mathrm{O}_{\text {handover }}$ MN the overhead of Mobile gateway switch and Mobile node switch respectively.

In this strategy, a handover is performed if the new gateway is closer than the old gateway. To determine whether a new gateway is closer than the old gateway, Euclidean distance is used. Let $\mathrm{D}_{\text {old }}$ be the distance of a node to its current gateway and $\mathrm{D}_{\text {new }}$ the distance of the node to a new mobile gateway. Then, $\mathrm{O}_{\text {detection }}$ can be expressed by the following equation.

$$
\mathrm{D}_{\text {new }}+\mathrm{a}=\mathrm{D}_{\text {old }}
$$

Where, ' $a$ ' is an integer constant.

$\mathrm{O}_{\text {execution }}$ is nothing but $\mathrm{O}_{\text {register }}$ with the new gateway. The handover is actually executed when following condition holds true for one agent advertisement.

$$
\mathrm{D}_{\text {new }}<\mathrm{D}_{\text {old }}
$$

\section{E. An Efficient Bi-Directional Connectivity Framework for Integrating MANET and Internet}

1) Description of the Bi-Directional Connectivity Framework (Khan et al., 2009)

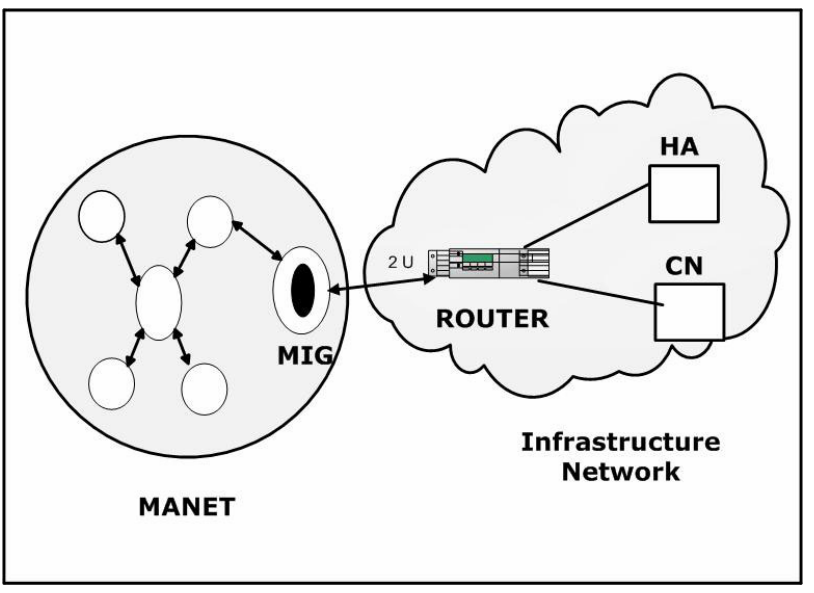

Figure 7. Basic Model of the Connectivity Framework

The objective of the bi directional connectivity framework is to integrate the ad hoc Network and the infrastructure (wired) network by means of the mobile hosts, which are located under the radio range of Mobile Internet Gateway

(MIG), which acts as the bridge between the hybrid networks. The ad hoc routing protocol Eff-DSDV (Khaleel et al., 2008d) and Mobile IP coordinate with each other to provide the connectivity across the hybrid network as shown in the Figure 7.

Analyzi There are two ways for the mobile host to obtain the global connectivity. In the first scenario, the mobile host may be directly under the coverage of the MIG i.e. one hop away from MIG, be able to send its data directly to the MIG. In the second case, the ad hoc hosts which are outside the range of the MIG communicate with the MIG using multi-hop communication links. One of the important objectives of the framework is to minimize the overhead of MIP and Eff-DSDV. Figure 6 shows the communication scenario between the two networks. The right side of the Figure shows the infrastructure network having the Home Agent, Correspondent node, MIG. The left side has the ad hoc hosts using the routing protocol. Every ad hoc host registers with the HA and creates a binding at HA by mapping its home address to the care of address (COA) provided by the MIG. The MIG acts as the FA for all the ad hoc hosts. After receiving the data packets sent by the $\mathrm{CN}$, the router tunnels them to the MIG/FA. Then the MIG delivers the packets to the ad hoc host using the Eff-DSDV protocol. On the other hand, after receiving the data packets sent by the ad hoc host, the MIG delivers them to the $\mathrm{CN}$ thru the router using the IP routing in the infrastructure network. In the proposed framework, MIG plays the role of FA. The MIG takes part in routing just as other Ad Host do. It also acts as Mobile IP Proxy for them. The MIG doesn't broadcast the Agent advertisements for the purpose of registration of the hosts. In the proposed protocol, ad hoc hosts and the MIG know each others presence via routing update of the Eff-DSDV protocol. Whenever the Ad hoc host say "A" joins the ad hoc network, the host A broadcasts DSDV advertisements to its neighbors with sequence number of ' 0 '. Each host takes a note of it and makes an entry about host " $A$ " in their routing tables. Later they broadcast with increased sequence number to their neighbors. This broadcasting process continues until the advertisements reach all the destinations i.e the diameter of the network. The MIG also comes to know about the host "A" and makes an entry in its routing table. The host " $\mathrm{A}$ " also gets routing updates from its neighbors and thereby creates its routing table, including the route to MIG. Thus all the nodes know routes to every other node in the network including the MIG. After this, the host "A" sends its registration information to the MIG like its Home Address. Based on this information MIG acts as a mobile IP proxy for " $\mathrm{A}$ ". The MIG sends the registration request to the host's Home Agent. After successful registration, a registration reply is returned to the MIG from the HA. The MIG then informs the host " $A$ " about the registration status. The MIG keeps the registration information of all the ad hoc hosts and uses it during re-registration. The MIG uses its address as the COA to register with the HA. The mobile IP registration lifetime is taken as 3 times that of DSDV periodic route update interval. The advantage of the proposed strategy is that the ad hoc host doesn't have to participate in the mobile IP registration, because MIG keeps all the registration information for each node. The MIG updates the registration for each ad hoc host before its expiration only if the MIG has a valid route to the ad hoc host. Therefore the MIG has the complete and consistent view of the entire network. It knows whether or not an ad hoc host is part of the network or not. In case if any ad hoc node roams away, then the hop count metric for the ad hoc host becomes infinite in the routing table of MIG. If it loses the route to an ad hoc host for 3 times the period of the EffDSDV update interval, then the MIG sends Deregistration message to the HA of the roamed away host. If the host joins the network again, then the MIG initiates a fresh registration process.

In order to be able to communicate with both network types, the MIG uses the protocols of the wired network and the wireless ad hoc Network. The ad hoc node uses the protocol stack that is similar to the wired nodes except 
that it uses Eff-DSDV protocol to route packets. To commission two protocol stacks, MIG uses two wireless interfaces. The first one is configured for the infrastructure mode and has the protocol stack of normal wired nodes. With this interface the MIG is able to connect to the Access Point. This Access Point is the connection between wireless and wired communication. It converts from wired to wireless and the other way round. The other interface of the MIG is configured for the ad hoc mode and has the Eff-DSDV ad hoc routing protocol

\section{2) Analyzing the Bi-Directional Connectivity Framework}

$\mathrm{O}_{\mathrm{BI}-\mathrm{DIR}}=\mathrm{Sum}$ of the overheads of the modules as stated in Equation (1)

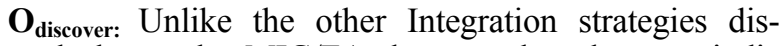
cussed above, the MIG/FA does not broadcast periodic Agent Advertisements and neither do the mobile nodes send Agent Solicitation messages to the MIG. The MIG is discovered by the mobile nodes by means of periodic exchange of routing tables. The gateway discovery over head is actually the overhead of the routing protocol. Once a mobile node discovers the MIG and the MIG comes to know the existence of the mobile node, both make an entry in their respective routing tables. The gateway discovery process is an automatic outcome of the routing protocol and no special messages are required. Therefore,

$$
\mathrm{O}_{\text {discover }}=0
$$

O $_{\text {register }}$ : Whenever a mobile node desires Internet connectivity, it registers itself with the MIG by unicasting a Registration request. The registration overhead is given by,

\section{NGM Si,register}

$$
\mathrm{O}_{\text {register }}=\sum_{\mathrm{i}=1} \sum_{\mathrm{j}=1} \quad\left[\begin{array}{r}
\mathrm{P}_{\text {REG_REQ }}+ \\
\mathrm{P}_{\text {REG_REP }}
\end{array}\right.
$$

Where,

$\mathrm{Si}$,register is the size of the RP selected to register with the gateway.

$\mathrm{P}_{\text {REG REQ }}$ and $\mathrm{P}_{\text {REG REP }}$ are the sizes of the Registration Request and Registration Reply packets respectively.

NGM is the set of all Gateway-Mobile Node pairs in the ad hoc network.

$\mathbf{O}_{\text {renewal }}$ : The renewal module is given by,

$$
\mathrm{O}_{\text {renewal }}=\sum_{\mathrm{i}=1} \sum_{\mathrm{j}=1}\left[\mathrm{P}_{\text {REG_REQ }}+\text { P }_{\text {REG_REP }}\right]
$$

Where, NGM, P PEG_REQ , $\mathrm{P}_{\text {REG_REP }}$ have the same meaning as in the registration module. $\mathrm{S}_{\mathrm{i} \text {,renewal }}$ is the size of the RP selected to renew the registration with the gateway.

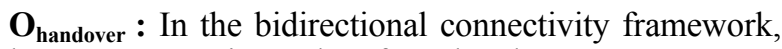
only one MIG exists. Therefore, handovers are not supported and the overhead involved is nil.

$$
\mathrm{O}_{\text {handover }}=0
$$

\section{F. Hierarchical Approach of Integrating MANET and the Internet}

1) Overview of the Hierarchical Approach of Integrating Mobile ad hoc Network and the Internet (Khan et al., 2008c)
In this Integration strategy, the Cluster-Head Gateway Switch Routing Protocol is integrated with Mobile IP to provide Internet connectivity to MANET nodes. This Integration strategy uses the Cluster-Head Gateway Switch Routing Protocol (CGSR) for routing within the MANET. The CGSR uses a hierarchical network topology, unlike other table driven routing approaches that employ flat topologies. CGSR organizes nodes into Clusters, with coordination among the members of each Cluster entrusted to a special node named Cluster Head $(\mathrm{CH})$. Clustering provides a mechanism to allocate bandwidth, which is a limited resource, among different Clusters, thereby improving reuse. CGSR assumes that all communication passes through the Cluster Head. Communication between two Clusters takes place through the common member nodes that are members of both the Clusters. These nodes which are members of more than one Cluster are called Cluster Gateway (CG). A Cluster Gateway is expected to be able to listen to multiple spreading codes that are currently in operation in the Clusters in which the node exists as a member. Figure 8 illustrates the inter Cluster communication by means of Cluster Gateway. In the Figure Mobile Node (MN1) sends the data to Mobile Node (MN2) first thru the Cluster Head then thru Gateway to Cluster Head of the destination Cluster and finally the packets are delivered to the destination mobile node.

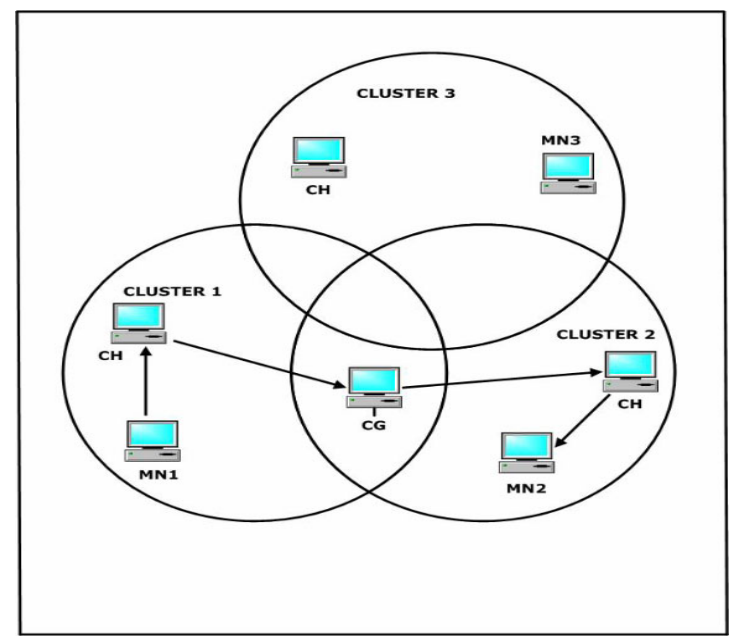

Figure 8. Mobile Node MN 1 communicating with Mobile Node MN 2 using CGSR.

The Integration architecture shown in Figure 9 consists of the Mobile ad hoc Network (MANET), with Clusters either overlapped or non-overlapped. Each Cluster has a Cluster Head and the overlapping Clusters have a Cluster Gateway. At least one Cluster Head shall be in the transmission range of the Foreign Agent (FA) in the Internet backbone. The Correspondent Node $(\mathrm{CN})$ is assumed to be present in the wired Internet.

The Integrated Protocol uses the basic functionalities of the Mobile IP as well as CGSR. It is assumed that the CHs are close to FA in order to provide the Internet connectivity to the MANET nodes. The $\mathrm{CHs}$ are assumed to be registered with some FA at any time. The Agent advertisements issued by the FA are meant only for the CHs. They are ignored by the ad hoc hosts and CGs. A proactive approach of registration of the $\mathrm{CH}$ with the $\mathrm{FA}$ is used. If any $\mathrm{CH}$ receives advertisements from multiple FAs, then it selects that FA which is lightly loaded. All the communication from the MANET nodes towards the 
Internet side is through the $\mathrm{CH}$. Therefore the $\mathrm{CHs}$ acts like a Mobile IP proxy for the MANET mobile nodes and the visiting mobile nodes.

The CH doesn't broadcast the Agent advertisements for the purpose of registration of the hosts. In this protocol, ad hoc hosts and the CHs know each others presence via routing update of the DSDV protocol, as the DSDV is the underlying protocol in the CGSR.

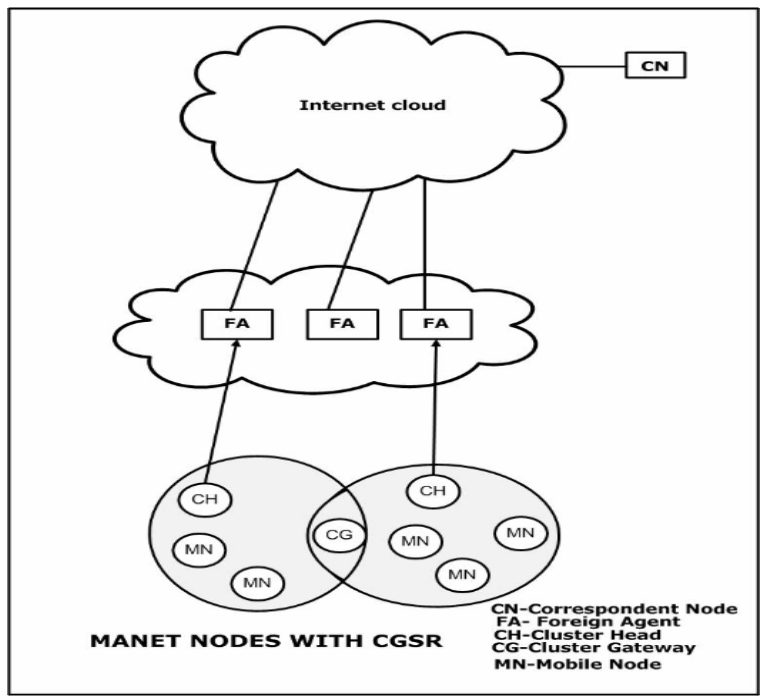

Figure 9. Hierarchical Architecture

Whenever the Ad hoc host say " $A$ " joins the ad hoc network, the host A broadcasts DSDV advertisements to its neighbors with sequence number of ' 0 '. Each host takes a note of it and makes an entry about host " $A$ " in their routing tables. Later they broadcast with increased sequence number to their neighbors. This broadcasting process continues until the advertisements reach all the destinations i.e. the diameter of the network. The $\mathrm{CH}$ also comes to know about the host " $\mathrm{A}$ " and makes an entry in its routing table. The host " $\mathrm{A}$ " also gets routing updates from its neighbors and thereby creates its routing table, including the route to $\mathrm{CH}$. Thus all the nodes know routes to every other node in the network. After this, the host "A" sends its registration information to the $\mathrm{CH}$ like its Home Address. Based on this information $\mathrm{CH}$ acts as a mobile IP proxy for "A". The $\mathrm{CH}$ sends the registration request to the host's Home Agent. After successful registration, a registration reply is returned to the $\mathrm{CH}$ from the $\mathrm{HA}$. The $\mathrm{CH}$ then informs the host " $\mathrm{A}$ " about the registration status. The $\mathrm{CH}$ keeps the registration information of all the ad hoc hosts and uses it during re-registration. The $\mathrm{CH}$ uses its address as the COA to register with the HA. The mobile IP registration lifetime is taken as 3 times that of DSDV periodic route update interval.

\section{2) Analyzing the Hierarchical approach}

$\mathrm{O}_{\text {HIERARCHICAL }}=$ Sum of the overheads of the modules as stated in Equation (1).

$\mathbf{O}_{\text {discover: }}$ Gateway discovery is of two types. Type one is the discovery of Internet Gateways/Foreign Agents by Cluster Heads and type two is the discovery of Cluster Head by mobile nodes. Therefore, the discovery overhead is divided into two sub components, the overhead of discovering the Internet Gateway $\left(\mathrm{O}_{\text {discover IG }}\right)$ and the overhead of discovering the Cluster Head $\left(\mathrm{O}_{\text {discover_CH }}\right)$. The total discovery overhead is given by:-

$$
\mathrm{O}_{\text {discover }}=\mathrm{O}_{\text {discover_IG }}+\mathrm{O}_{\text {discover_CH }}
$$

In the discovery of Internet Gateway, a proactive approach is used. The $\mathrm{O}_{\text {discover_IG }}$ is given by

$$
\mathrm{O}_{\text {discover_IG }}=\sum_{\mathrm{i}=1} \begin{array}{rr}
\sum_{\mathrm{j}} \mathrm{P}_{\mathrm{AA}} \\
\mathrm{j}=1
\end{array}
$$

Where,

NGC is the number of Internet Gateway-Cluster Head pairs, $\mathrm{S}_{\mathrm{i}, \mathrm{RPS}}$ is the size of the RPS of the ith Internet Gateway-Cluster Head pair, $\mathrm{P}_{\mathrm{AA}}$ is the size of the Agent advertisement packet.

The DSDV routing protocol is used for routing within the ad hoc network. The Cluster Heads are discovered by the mobile nodes due to the periodic exchange of routing tables. No additional messages are required to discover the Cluster Heads. Therefore,

$$
\mathrm{O}_{\text {discover_CH }}=0
$$

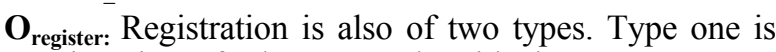
the registration of Cluster Heads with the Internet Gateway and type two is the registration of mobile nodes with Cluster Head. Therefore, the registration overhead is given by,

$$
\mathrm{O}_{\text {register }}=\mathrm{O}_{\text {register_IG }}+\mathrm{O}_{\text {register_CH }}
$$

Where, $\mathrm{O}_{\text {register_IG }}$ and $\mathrm{O}_{\text {register_MG }}$ are the respective overheads of registering with the Internet Gateway and Cluster Head.

$$
\begin{aligned}
& \mathrm{NICH} \quad \mathrm{S}_{\mathrm{i}, \text { register }} \\
& \mathrm{O}_{\text {register_IG }}=\sum \sum\left[\mathrm{P}_{\mathrm{REG}_{-} \mathrm{REQ}}{ }^{+}\right. \\
& \left.\mathrm{i}=1 \quad \mathrm{j}=1 \quad \mathrm{P}_{\text {REG_REP }}\right] \\
& \text { NCHM } \mathrm{S}_{\mathrm{i}, \text { register }} \\
& \mathrm{O}_{\text {register_CH }}=\sum_{\mathrm{i}=1} \quad \sum_{\mathrm{j}=1}\left[\mathrm{P}_{\text {REG_REQ }}{ }^{+} \mathrm{P}_{\text {REG_REP }}\right]
\end{aligned}
$$

Where NICH is the number of Internet GatewayCluster Head pairs, and NCHM is the number of Cluster Head- Mobile Node pairs in the ad hoc network.

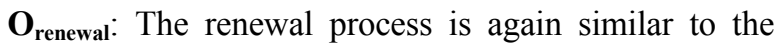
registration process, the only difference being that the $\mathrm{Si}$,register may or may not be the same as Si,renewal .

$$
\mathrm{O}_{\text {renewal }}=\mathrm{O}_{\text {renewal_IG }}+\mathrm{O}_{\text {renewal_CH }}
$$

Where, $\mathrm{O}_{\text {renewal IG }}$ and $\mathrm{O}_{\text {renewal } \mathrm{CH}}$ are the overhead of renewal with the Internet Gateway and Cluster Head respectively.

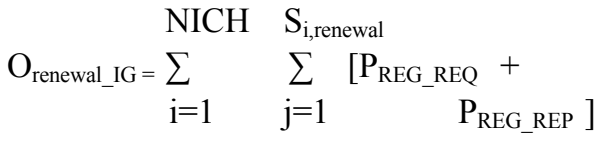

$$
\begin{aligned}
& \left.\mathrm{O}_{\text {renewal_CH }}=\sum_{\mathrm{i}=1} \sum_{\mathrm{j}=1} \mathrm{P}_{\text {REG_REQ }}+\mathrm{P}_{\text {REG_REP }}\right]
\end{aligned}
$$

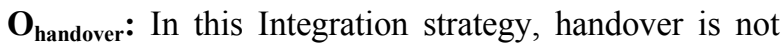
currently supported. Therefore,

$$
\mathrm{O}_{\text {handover }}=0
$$




\section{AN ANALYTICAL FRAMEWORK FOR THE ASSESSMENT OF THE MOBILE IP OVERHEAD INVOLVED IN THE INTEGRATED INTERNET-MANET}

V. SimUlations AND COMPARISON OF THE INTEGRATION STRATEGIES USING THE ANALYTICAL FRAMEWORK

The proposed analytical model has been implemented in a simulated environment based on a sample topology of two gateways and 5 mobile nodes, resulting in 10 Gateway-Mobile Node pairs. The simulation was developed in $\mathrm{C}++$. Figure 10 shows the comparison of the overall control overhead of MIPMANET, Sun et al and BiDirectional Connectivity Framework. We can observe the higher overhead of Sun et al's strategy, as predicted by the analytical model.

We used a sample topology of two Internet gateways, three Mobile gateways and five mobile nodes to simulate the Ammari et al strategy and the Hierarchical approach. Figure 11 shows the comparison of these two strategies. We observe that Ammari et al 's strategy incurs a higher overhead when compared to the Hierarchical approach, as predicted by the Analytical Model.

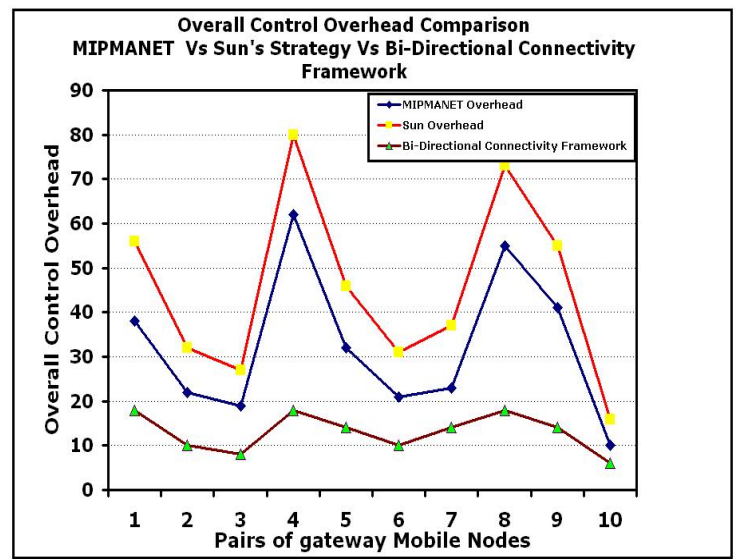

Figure 10. Comparison of overall control Overhead of MIPMANET, Sun et al's Strategy and the Bi-Directional Connectivity Framework

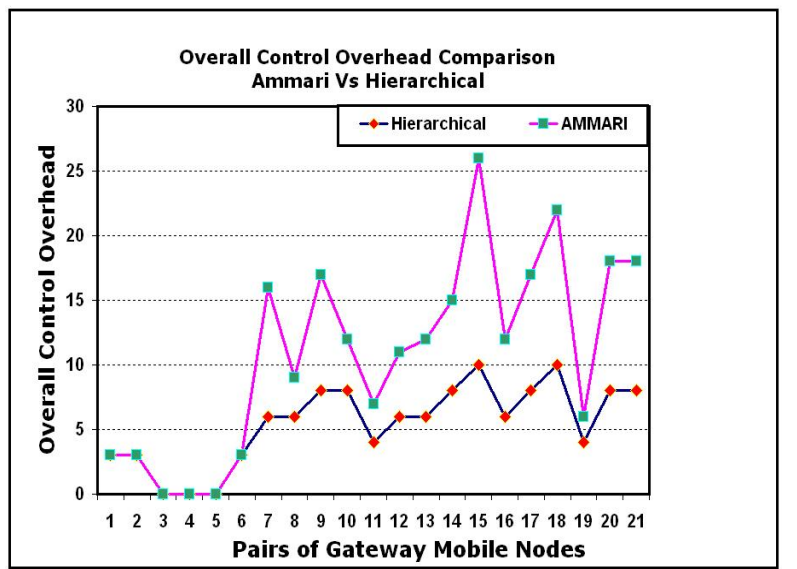

Figure 11. Comparison of overall control Overhead of Ammari et al's Strategy and the Hierarchical Approach

The analytical model can be used to analyze the performance of an Integration strategy by substituting one protocol module for another. To demonstrate this aspect, we have substituted the proactive gateway discovery mechanism of MIPMANET with a reactive one. Figure 12 shows the performance comparison of proactive and reactive approaches of gateway discovery in MIPMANET. The Figure once again demonstrates that the proactive gateway discovery mechanism requires a higher overhead when compared to a reactive gateway discovery mechanism.

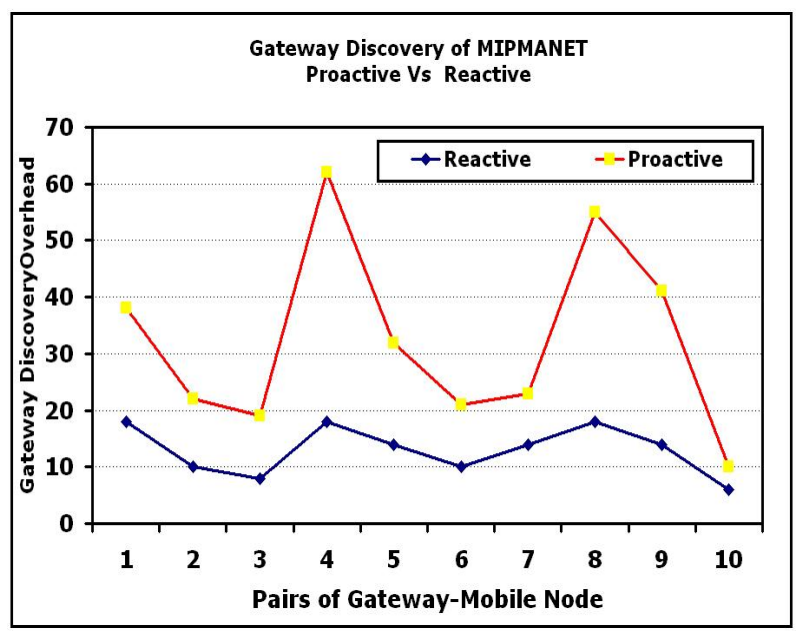

Figure 12. Performance comparison of Proactive and Reactive approaches for Gateway Discovery in MIPMANET.

\section{CONCLUSIONS}

In this paper, an analytical model based on the concept of a Relay Path which can represent, characterize and compare the Integration Strategies of Mobile Ad Hoc Networks and the Internet has been proposed. The same has been applied to five of the existing Integration Strategies. The performance comparison has been done by simulating the strategies based on the analytical framework by taking a sample topology. The analytical framework demonstrates that it is much easier to assess the control overhead of the strategies and make a performance comparison among them to find out the most efficient one. It is intended to bring out taxonomy of the Integration Strategies based on the proposed model. The framework can also be used to suggest improvements and also propose new strategies. In future, the Analytical model can be extended to make it more generalized by taking into other metrics like power consumption, Temporal metrics etc.

\section{REFERENCES}

[1] Ammari, H. and El-Rewini, H., (2004) 'Integration of Mobile Ad Hoc Networks and the Internet Using Mobile Gateways', Proceedings of the $18^{\text {th }}$ International Parallel and Distributed Processing Symposium (IPDPS '04) Workshop 12, pp 218 b.

[2] Broch, J., Maltz, D.A. and Johnson, D.B., (1999) 'Supporting Hierarchy and Heterogeneous Interfaces in Multi-Hop Wireless Ad Hoc Networks', Proceedings of Fourth International Symposium on Parallel Architectures, Algorithms, and Networks (I$S P A N^{\prime}$ '99), Fremantle, Australia, pp 370-375.

[3] Jing N., Zeng, L. and Wen, J., (2007) 'Handoffs in Integrating Ad Hoc Networks to Internet', Proceedings of International Conference on Convergence Information Technology, IEEE Computer Society, pp 1378-1383.

[4] Johnson, D., Perkins, C. and Arkko, J., (2004) 'Mobility Support in IPv6', RFC 3775, June.

[5] Jonsson, U., Alriksson, F., Larsson, T., Johansson, P. and Maguire, G.Q Jr., (2000) 'MIPMANET - Mobile IP for Mobile Ad Hoc Networks', The First IEEE/ACM Annual Workshop on Mobile Ad Hoc Networking and Computing(MobiHOC), Boston, Massachusetts, USA, August 11, pp 75-85.

[6] Khan K.R., Rafi U, Zaman. and Venugopal, Reddy A., (2008) 'Integrating Mobile Ad Hoc Networks and the Internet: challenges and a review of strategies', Communication Systems Software and 
Middleware and Workshops COMSWARE 2008, 3rd International Conference, 6-10 Jan, pp 536-543.

[7] Khan K.R., Rafi U, Zaman. and Venugopal, Reddy A., (2008) 'An Efficient DSDV Routing Protocol for Wireless Mobile Ad Hoc Networks and its Performance Comparison', Second UKSIM European Symposium on Computer Modeling and Simulation , 10-12,September, England. pp. 506-511

[8] Khan K.R, Rafi U, Zaman. and Venugopal, R.A., (2008) 'A Three-Tier Architecture for Integrating Mobile Ad Hoc Network and the Internet using a Hierarchical Integrated Routing Protocol', Intl conference on advanced computer theory and engineering, 2224 December, Thailand.

[9] Khan K.R, Rafi U, Zaman. and Venugopal, R.A., (2008) 'A BiDirectional Connectivity Framework for Mobile Ad Hoc Network and the Internet', IFIP Wireless days Conference, 24-27 November, UAE.

[10] Khan, K.R., Zaman, R.U. and Reddy, A.V. (2009) “An Efficient Integrated Routing Protocol for Interconnecting Mobile Ad Hoc Network and the Internet", ICAC3'09, Mumbai, January 2009, In the proceedings of ACM, ISBN number - 978-1-60558- 351-8

[11] Lei, H. and Perkins, C.E., (1997) 'Ad Hoc Networking with Mobile IP', Proceedings of the $2^{\text {nd }}$ European Personal Mobile Communications Conference, Bonn, Germany, pp 197-202.

[12] Perkins, C.E, (1997)“ Mobile IP,' IEEE Communications Magazine, 3(5), pp 84-99. (doi:10.1109/35.592101)

[13] Perkins, C.E. and Royer, E.M., (2002) ' Ad-Hoc on Demand Distance Vector Routing', Internet Engineering Task Force, Internet Draft, June

[14] Ratanchandani, P. and Kravets, R., (2003) 'A Hybrid Approach to Internet Connectivity for Mobile Ad Hoc Networks', Proceedings of IEEE Wireless Communications and Networking, New Orleans, Louisiana, pp1522-1527.

[15] Sun, Y.,Belding-Royer, E.M. and Perkins, C.E., (2002) 'Internet Connectivity for Ad Hoc Mobile Networks', International Journal of Wireless Information Networks, Special Issue on Mobile Ad Hoc Networks(MANETs):Standards, Research, Applications, April.
[16] Tao, L., Midkiff, S.F. and Park, J.S., (2003) 'A framework for wireless Ad Hoc routing protocols', IEEE Wireless Communications and Networking, 2003, Volume 2, Issue , 16- 20 March.

[17] Tseng, Y., Shen, C. and Chen, W. (2003) ' Integrating Mobile IP with Ad Hoc Networks', IEEE Computer, 36(5), pp 48-55.

\section{AUTHORS}

Dr. Khaleel Ur Rahman Khan obtained B.E. (CSE) from Osmania University in 1993, M.Tech (CS) from JNTU in 1998, Ph D in CS from O.U in June 2009. He is presently working as Professor and Head CSE Department at Muffakham Jah College of Engineering and Technology. His research interests include Routing and Multicasting in Ad Hoc Networks.

Rafi U Zaman was born in Hyderabad, India, on May 19, 1979. He obtained the B.Sc. (Computer Science) and M.C.A. degrees from Osmania University in 1999 and 2002 respectively. $\mathrm{He}$ is currently pursuing M.Tech (CSE), Part-Time, from Osmania University and working as an Assistant Professor in Muffakham Jah College of Engg. And Tech, Hyderabad. His research interest is Integration of Mobile Ad Hoc Network with the Internet.

Dr. A. Venugopal Reddy has done his M.Tech. from IIT Delhi in 1979 and Ph.D. from University of Roorkee (IIT Roorkee) in the year 1994. He has a total of 27 years of teaching experience, teaching to both undergraduate and graduate students. Currently he is working as Professor in Computer Science and Engineering Department at the College of Engineering, Osmania University. He is also Dean, Faculty of Informatics at O.U. His current subject interests include Parallel Algorithms, Computer Networks, Mobile Ad hoc Networks.

Submitted 30 July 2009. Published as resubmitted by the authors on December, 2, 2009. 